\title{
Crystallography at the Nanoscale: t-EBSD Study of npAu Catalysts
}

Cédric Barroo ${ }^{1,2,3}$, Austin J. Akey ${ }^{4}$ and David C. Bell ${ }^{2,4}$

1. Chemical Physics of Materials and Catalysis, Université libre de Bruxelles, Brussels, Belgium.

2. John A. Paulson School of Engineering and Applied Sciences, Harvard University, Cambridge, MA.

3. Department of Chemistry and Chemical Biology, Harvard University, Cambridge, MA.

4. Center for Nanoscale Systems, Harvard University, Cambridge, MA.

In recent years, there has been a growing interest in the use of self-supported catalysts in the form of nanoporous bulk materials since they combine unique properties important for applied formulations of catalysts. At the same time, current research focusses on the development of catalysts efficient at low temperature for which gold appears to be a good candidate, especially for selective oxidation reactions. The two main parameters dictating the reactivity of gold catalysts are the size of the nanoparticles and the nature of the support: it is indeed known that large $\mathrm{Au}$ nanoparticles (size $>10 \mathrm{~nm}$ ) are not catalytically active. To get rid of this size-dependence, alloying gold with less noble transition metals seems to be very promising. The idea is to combine a minor amount of active metal that facilitates creation of reactive intermediates with a less active majority phase that transforms these intermediates to desirable products, with high selectivity. It is then possible to fine tune the catalytic performance (activity and selectivity) of the Au-based materials by using different concentration of the minor active metal.

In this context, nanoporous gold catalysts (npAu) are currently used for selective alcohol oxidation reactions. These catalysts are prepared by dealloying of a bulk Au-Ag alloy, and result in a porous structure with a composition of $\mathrm{Au}_{97}-\mathrm{Ag}_{3}$. Morphological and compositional changes may occur under reaction conditions and can dramatically influence the reactivity and/or selectivity. Especially since the npAu is first activated under ozone atmosphere to confer its high catalytic activity [1]. In order to improve both the efficiency and selectivity, it is crucial to understand the behavior of Ag during the reaction and to control the micro- and nanostructure of the catalyst.

In the present work, we studied the grain morphology of nanoporous Au catalyst by transmissionElectron BackScatter Diffraction (t-EBSD). t-EBSD experiments were performed (Figure 1.a) on a FEI Helios Nanolab 660 with an EDAX EBSD system and TEAM software. The electron beam was set to $30 \mathrm{kV}$ and 6-13 nA, with a working distance of approximately $4 \mathrm{~mm}$. Previously, this technique has been successfully used for atom probe tomography applications on samples of similar sizes [2]. Transmission-EBSD was here used to study the nano-crystallography of npAu. The ligament size of those samples is in the 50-70 nm range, and the porosity of the materials corresponds to $70 \%$. Samples were prepared as "mono-ligaments thin" lamellae using the lift-out method (Figure 1.b).

The high resolution of the t-EBSD allows probing the crystallography of npAu with decreasing steps of $8 \mathrm{~nm}$ (Figure 2.a-b), $5 \mathrm{~nm}$ and even $2 \mathrm{~nm}$. In all the cases, the quality of the pattern diffraction is sufficient to undoubtedly assign a crystallographic orientation to the sample (Figure 2.c). By analyzing large areas, we proved the monolithic nature of the sample, down to the nanoscale: this is important from a synthesis point of view since there is a retention of the crystallography during the dealloying process. With a step size of $2 \mathrm{~nm}$, the quality of the analysis decreases due to the presence of vibrations. However, with such resolution, it should be possible to observe the formation of small clusters $(>5 \mathrm{~nm})$ 
of $\mathrm{Ag}$ or $\mathrm{AgO}_{\mathrm{x}}$ after ozone treatment. As a conclusion, this t-EBSD study revealed the retention of grain structure during dealloying of Au-Ag alloy down to the nanoscale, as well as the high resolution of this technique and its potential applications to changes in the crystallography of nanoporous materials [3].

\section{References:}

[1] C. Barroo et al, J. Phys. Chem. C 121 (2017), p. 5115.

[2] K. Babinsky et al, Ultramicroscopy 144 (2014), p. 9.

[3] This work was supported as part of the Integrated Mesoscale Architectures for Sustainable Catalysis - IMASC, an Energy Frontier Research Center funded by the U.S. Department of Energy, Office of Science, Basic Energy Sciences under Award \# DE-SC0012573. C.B. acknowledges postdoctoral fellowships through the Belgian American Educational Foundation (BAEF) as well as WallonieBruxelles International (Excellence grant WBI.WORLD) foundations.
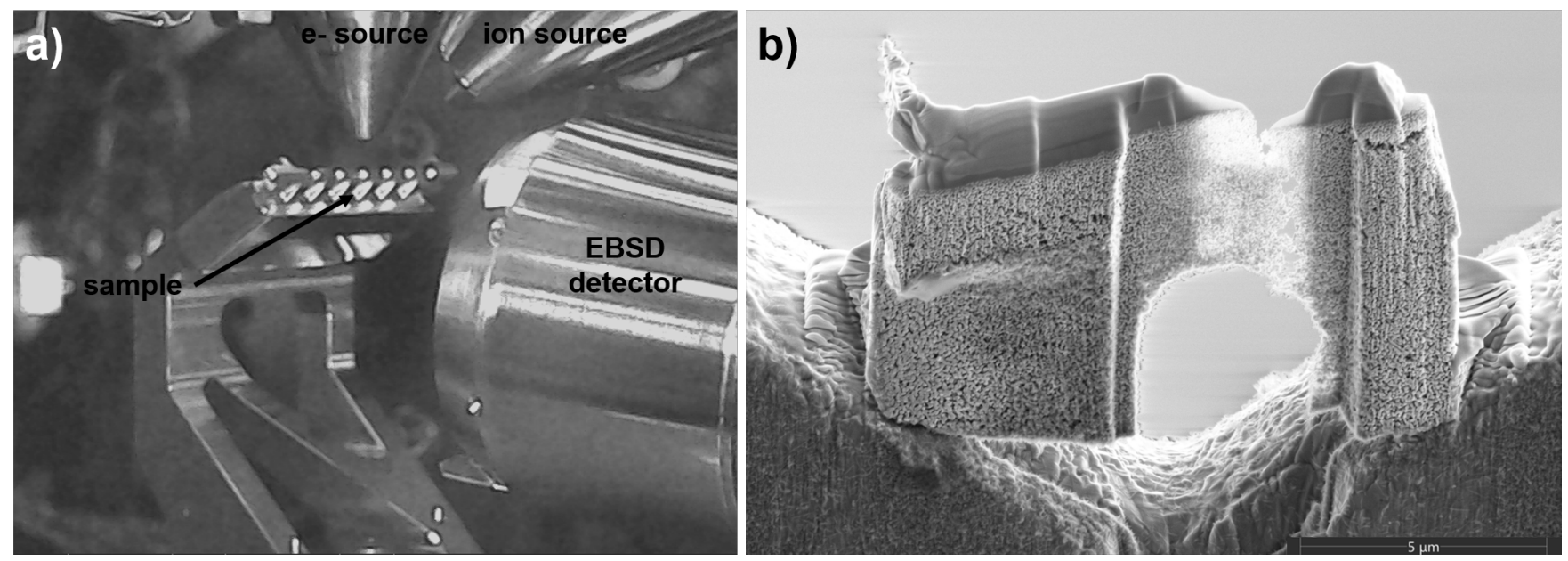

Figure 1. a) Inside view of the system during t-EBSD experiments; b) Prepared npAu sample.
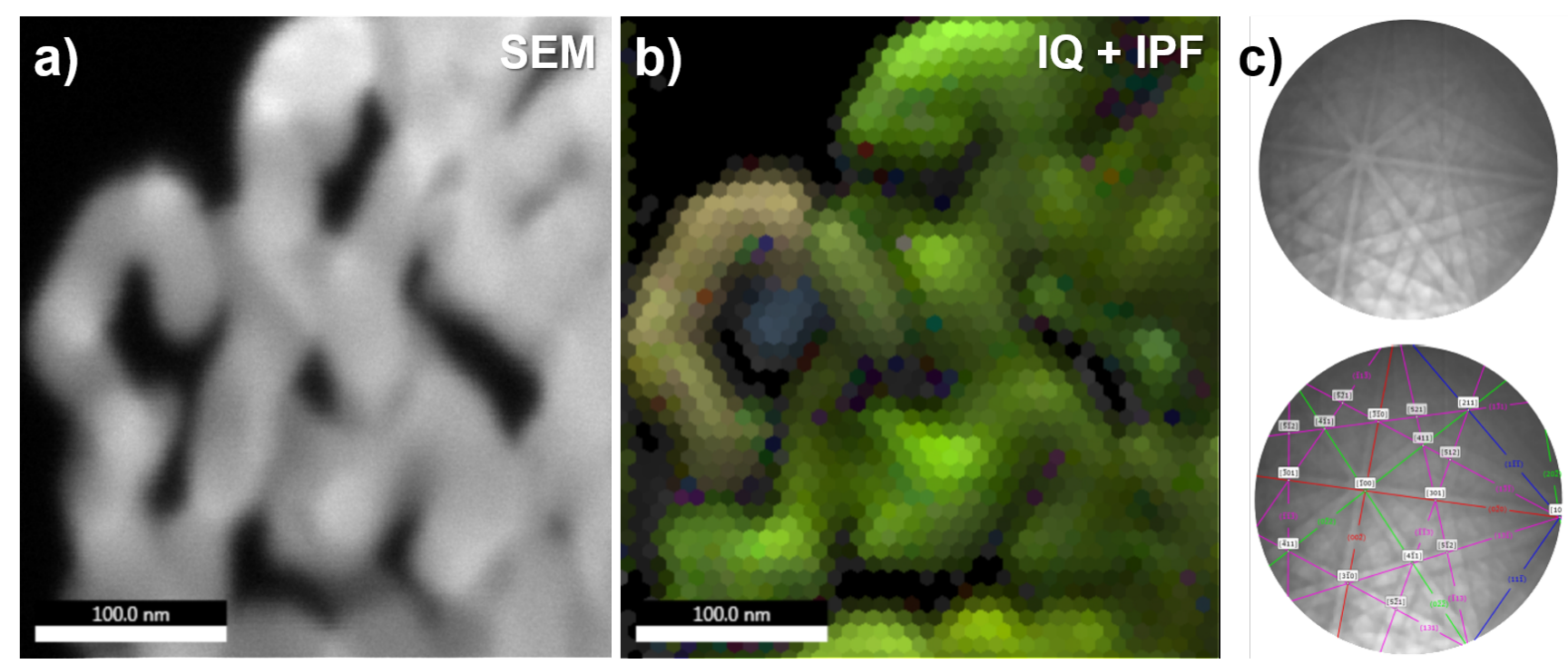

Figure 2. a) SEM image of a "mono-ligaments thin" lamellae of npAu; b) t-EBSD analysis: superposition of Image Quality (IQ) and Inverse Pole Figure (IPF) images showing the monolithic nature of the sample; c) Diffraction pattern and indexation. Step size for the analysis: $8 \mathrm{~nm}$. 